\title{
1 A bootstrap approach is a superior statistical method for the comparison of cell-to-cell movement \\ 2 data
}

3 Matthew G. Johnston ${ }^{1}$ and Christine Faulkner

4 Department of Crop Genetics, John Innes Centre, Norwich, UK

$5 \quad{ }^{1}$ Corresponding author: matthew.johnston@jic.ac.uk, $+44(0) 1603450000$

6 Total words: 1176

7 Total figures: 1

\section{Keywords}

9 Plasmodesmata, bootstrap, Mann-Wilcox-Whitney, cell-to-cell movement, statistics

10 Summary

11 Plasmodesmata are an increasing focus of plant research, and plant physiologists frequently aim to

12 understand the dynamics of intercellular movement and plasmodesmal function. For this,

13 experiments that measure the spread of GFP between cells are commonly performed to indicate

14 whether plasmodesmata are more open or closed in different conditions or in different genotypes.

15 We propose cell-to-cell movement data sets are better analysed by a bootstrap method that tests

16 the null hypothesis that means (or medians) are the same between two conditions, instead of the

17 commonly used Mann-Whitney-Wilcoxon test. We found that that with hypothetical distributions

18 similar to cell-to-cell movement data, the Mann-Whitney-Wilcoxon produces a false positive rate of

$1917 \%$ while the bootstrap method maintains a false positive at the set rate of $5 \%$ under the same

20 circumstances. Here we present this finding, as well as our rationale, an explanation of the

21 bootstrap method and an R script for easy use. We have further demonstrated its use on published

22 datasets from independent laboratories.

\section{Main Text}

24 Symplastic cell-to-cell connectivity is dynamically regulated in plants as a component of 25 developmental and environmental responses (Perbal et al., 1996; Wada et al., 2002; Faulkner et al., 26 2013). Connectivity is established between cells by plasmodesmata, which function as a key 27 parameter to define the dynamics of cell-to-cell connectivity. It is critical to assay the degree of 28 movement of different molecules between cells to understand the range and dynamics of cell-to-cell 29 communication as well as to assay plasmodesmal function under different conditions or in different 30 genotypes. Accurate experimental analysis is critical to understanding this important component of 31 plant physiology. 
32 There are two routinely used methods, with a cellular resolution, to assay the spread of GREEN

33 FLUORESCENT PROTEIN (GFP) from one cell into neighbouring cells: microprojectile bombardment,

34 and low $\mathrm{OD}_{600}$ Agrobacterium tumefaciens infiltration (Oparka et al., 1999; Burch-Smith \& Zambryski,

35 2010). These assays allow the experimenter to count the number of cells ('cell count'), or the

36 number of concentric rings of cells ('cell layers'), to which GFP has spread from a single cell (Fig. 1a).

37 This serves as a measure of symplastic connectivity - the further the GFP has spread, the greater the

38 degree of connection (or of plasmodesmata permeability) between cells. Neither cell nor layer counts are parametrically distributed (Fig. $\mathbf{1 b}-\mathbf{d}$, upper), so most studies use the non-parametric Mann-Whitney-Wilcoxon (MWW) test to compare conditions to identify factors that regulate the connection and communication between cells.

Most experiments aim to assess whether connectivity is greater or less under different conditions, or whether plasmodesmata are more open or closed, which involves analysis of changes in the median or mean. The MWW tests the null hypothesis that two data distributions are the same (Mann \& Whitney, 1947), not whether the two distributions have the same median. Therefore, it is possible to find a significant difference in an MWW test with distributions that have the same median, but different variances (Hart, 2001). When data from cell count assays are presented in histogram form, it is clear that the shape of the distributions differs between experimentally compared conditions or genotypes (Fig. 1b, c) (Guseman et al., 2010; Diao et al., 2018; Cheval et al., 2020). Thus, if an MWW test is used on cell count data, the difference in distribution shapes between conditions may lead to the erroneous conclusion that there is a significant difference in the amount of spread of GFP.

Therefore, a different statistical method is required to properly interpret differences in GFP spread. For this, we propose a bootstrap method (Efron, 1979). Unlike the MWW test, bootstrapping works with data that is both non-parametric and heteroskedastic (differing variance between conditions).

The goal of the analysis is to estimate the probability that the observed difference in medians $(\hat{\theta})$ came about by chance (a $p$ value). Frequentist statistics does this by comparing $\hat{\theta}$ to a null distribution. In this case, the null distribution describes the probability of observing a difference in medians, when there is no true difference in the underlying data. Usually, a known distribution is used (e.g. t-distribution or F-distribution) but in this case it is unknown because the data do not follow parametric distributions (Fig. 1b, c).

Bootstrapping techniques can be used to generate a null distribution de novo from the observed data already collected, as long as the samples are independent. This removes the requirement of using a known distribution. To do this, the observed data are sampled with replacement to generate 
population. The relationship between multiple resamples and the observed data can be used to reveal how the observed data relate to the true population, and so estimate a $p$ value for the observation.

An example $R$ function is provided to perform this analysis (medianBootstrap. $R$, https://github.com/faulknerfalcons/Johnston-2020-Bootstrap), which requires two arguments, i.e. two vectors of numbers: control and treatment. The function generates a null distribution to compare against by resampling each vector $N$ times (by default 5000) and, for each resample, generating a resampled test statistic $\left(\widehat{\theta^{*}}\right)$. These $N$ resampled test statistics are made into a null distribution by $\left|{\widehat{\theta^{*}}}_{n}-\hat{\theta}\right|$ (Fig. 1b - c, lower) as suggested by Hall and Wilson (1991).

As this is a random sampling technique, an exact $p$ value cannot be calculated but an estimate is produced: a Monte Carlo $\hat{p}$ value (Eqn. 1). To do so, $\hat{\theta}$ is compared to the null distribution to find the chance of observing a value at least as extreme (line on Fig. $\mathbf{1 b}-\mathbf{c}$, lower). $\mathrm{A}+1$ is added to the numerator and denominator in Eqn. 1 as suggested by Davison and Hinkley (1997): conceptually, this can be considered as including the observed sample among the bootstrap resamples.

$$
\hat{p}=\frac{\sum_{n=1}^{N} I\left(\left|\widehat{\theta}_{n}^{*}-\hat{\theta}\right| \geq \hat{\theta}\right)+1}{N+1} \quad \text { Eqn.1 }
$$

where $I(\cdot)$ is the indicator function.

80 As $\hat{p}$ is an estimate of $p$, a 95\% confidence interval should be constructed, where $p$ will fall within 81 this range $95 \%$ of the time (Wilson, 1927).

82 This method is not confounded by differences in variance or shape as with the MWW test. To 83 illustrate this, we compared the Type I error rate (false positives) between the MWW and medianBootstrap tests, when testing if there is a difference in medians between two populations for which there was no true difference in medians, i.e. $\theta=0$. In this scenario, an error rate of $5 \%$ is expected at $\alpha=0.05$. Samples $(n=100)$ for each population were drawn from normal distributions with the same variance $(X, Y \sim N(0,1))$ simulated in $\mathrm{R} 4.0 .0$ ( $\mathrm{R}$ Core Team, 2020). Both the MWW and medianBootstrap methods gave a difference in medians about $5 \%$ of the time, as expected $(4.5 \%(95 \% \mathrm{Cl}[3.4,6.0])$ and $4.9 \%(95 \% \mathrm{Cl}[3.7,6.4])$, respectively). When variances differed between populations $\left(X \sim N(0,1), Y \sim N\left(0,5^{2}\right)\right)$, the MWW test had a false positive rate significantly higher than the set $5 \%$ of $7.5 \%(95 \% \mathrm{Cl}[6.0,9.3])$. Conversely, the false positive rate of the medianBootstrap method was correctly controlled at $4.7 \%(95 \% \mathrm{Cl}[3.6,6.2])$.

93 Alternatively, when two samples are drawn from populations with equal variance and median, but 94 differing shape $\left(X \sim N\left(1-\frac{1}{\sqrt[3]{2}}, \frac{3}{80}\right), Y \sim \operatorname{Beta}(1,3)\right)$, a medianBootstrap method finds a significant 
difference in $5.1 \%$ of the trials $(95 \% \mathrm{Cl}[3.9,6.6])$, as expected. Whereas, an MWW test inflates the Type I error rate to $17 \%(95 \% \mathrm{Cl}[15,19])$. Therefore, as cell count data exhibit unequal variances and differing distribution shapes between conditions and/or genotypes, we propose that bootstrap methods are a more appropriate analysis to identify differences in the spread of GFP. It is worth noting that any test statistic, $\theta$, can be computed in a bootstrapped manner, provided the test is invariant to scaling. This means bootstrap testing can be extended to cell layer data, where means should be compared, as there is no difference in medians (Fig. 1d). An example of this extension is given in medianBootstrap.html.

We acknowledge alternative advanced statistical techniques, such as linear mixed effects models, for the analysis of these data. However, they require more assumptions and are less user-friendly. We consider this bootstrap method a good, easy-to-use, superior alternative to MWW analysis of cell-tocell movement data.

\section{Acknowledgments}

We thank Joanna Jennings (Department of Crop Genetics, John Innes Centre) for providing the confocal micrograph in Fig. 1a and Dr Joshua Hodgson (Department of Medicine, University of Cambridge) and $\operatorname{Dr}$ Matthew Castle (Department of Genetics, University of Cambridge) for constructive comments on the manuscript. The data in Fig. 1 comes from (b) Figure S2 of Cheval et al. (2020), (c) Figure 2d Diao et al. (2018), (d) Figure 2c Diao et al. (2018) under use of the CC BY 4.0 licence. MGJ is funded by a John Innes Foundation Studentship. Research in the Faulkner lab is supported by the Biotechnology and Biological Research Council Grant (BB/L000466/1, BBS/E/J/000PR9796) and the European Research Council (725459, “INTERCELLAR”).

\section{Author Contributions}

MGJ and CF designed, discussed and wrote up the research. MGJ performed the analysis.

\section{References}

Burch-Smith TM, Zambryski PC. 2010. Loss of increased size exclusion limit (ise)1 or ise 2 increases the formation of secondary plasmodesmata. Current Biology 20: 989-993.

Cheval C, Samwald S, Johnston MG, de Keijzer J, Breakspear A, Liu X, Bellandi A, Kadota Y, Zipfel C, Faulkner C. 2020. Chitin perception in plasmodesmata characterizes submembrane immunesignaling specificity in plants. Proceedings of the National Academy of Sciences 117: 9621-9629.

Davison AC, Hinkley D V. 1997. Bootstrap Methods and their Application. Cambridge University Press.

Diao M, Ren S, Wang Q, Qian L, Shen J, Liu Y, Huang S. 2018. Arabidopsis formin 2 regulates cell-tocell trafficking by capping and stabilizing actin filaments at plasmodesmata. eLife 7: e36316.

Efron B. 1979. Bootstrap Methods: Another Look at the Jackknife. The Annals of Statistics 7: 1-26. 
Faulkner C, Petutschnig E, Benitez-Alfonso Y, Beck M, Robatzek S, Lipka V, Maule AJ. 2013. LYM2dependent chitin perception limits molecular flux via plasmodesmata. Proceedings of the National Academy of Sciences 110: 9166-9170.

Guseman JM, Lee JS, Bogenschutz NL, Peterson KM, Virata RE, Xie B, Kanaoka MM, Hong Z, Torii KU. 2010. Dysregulation of cell-to-cell connectivity and stomatal patterning by loss-of-function mutation in Arabidopsis CHORUS (GLUCAN SYNTHASE-LIKE 8). Development 137: 1731-1741.

Hall P, Wilson SR. 1991. Two Guidelines for Bootstrap Hypothesis Testing. Biometrics 47: 757-762.

Hart A. 2001. Mann-Whitney test is not just a test of medians: Differences in spread can be important. British Medical Journal 323: 391-393.

Mann HB, Whitney DR. 1947. On a Test of Whether one of Two Random Variables is Stochastically Larger than the Other. The Annals of Mathematical Statistics 18: 50-60.

Oparka KJ, Roberts AG, Boevink P, Cruz SS, Roberts I, Pradel KS, Imlau A, Kotlizky G, Sauer N, Epel B. 1999. Simple, but not branched, plasmodesmata allow the nonspecific trafficking of proteins in developing tobacco leaves. Cell 97: 743-754.

Perbal MC, Haughn G, Saedler H, Schwarz-Sommer Z. 1996. Non-cell-autonomous function of the Antirrhinum floral homeotic proteins DEFICIENS and GLOBOSA is exerted by their polar cell-to-cell trafficking. Development 122: 3433-41.

R Core Team. 2020. R: A Language and Environment for Statistical Computing.

Wada T, Kurata T, Tominaga R, Koshino-Kimura Y, Tachibana T, Goto K, Marks MD, Shimura Y, Okada K. 2002. Role of a positive regulator of root hair development, CAPRICE, in Arabidopsis root epidermal cell differentiation. Development 129: 5409-19.

Wilson EB. 1927. Probable Inference, the Law of Succession, and Statistical Inference. Journal of the American Statistical Association 22: 209-212.

Figure 1 Bootstrap statistics on GFP movement data.

(a) An example image of GFP moving from a single transformation site. The degree of movement can either be counted as the number of fluorescent cells (denoted with stars, 17 cells) or the number of cell layers with GFP (blue overlays, 3 layers). Scale bar $=100 \mu \mathrm{m}$. (b - d) Top: Histogram of cell counts or layers, with the median and mean marked. Bottom: Bootstrap null distributions $\left(\left|\widehat{\theta^{*}}-\hat{\theta}\right|\right)$ for the differences in $(b, c)$ median or $(\mathbf{d})$ mean, with estimated $\hat{p}$ value and $95 \%$ confidence intervals $(\mathrm{Cl})$. The observed difference $(\hat{\theta})$ is marked by a red line. Data for (b) from Cheval et al. (2020) and data for (c, d) from Diao et al. (2018) both under the CC BY 4.0 licence. 

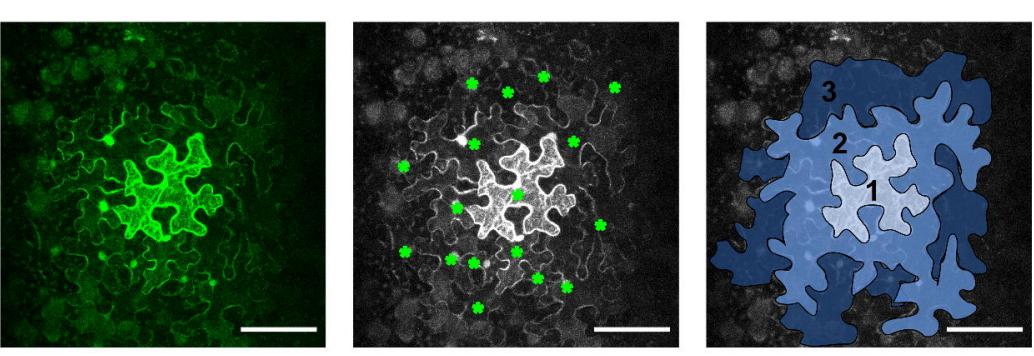

- - - Median

- Mean

$\theta \quad$ Test statistic

$\hat{\theta} \quad$ Sampled test statistic

$\hat{\theta^{*}} \quad$ Resampled test statistic

$\Delta_{\mu} \quad$ Difference in means

$\Delta_{\text {median }}$ Difference in medians
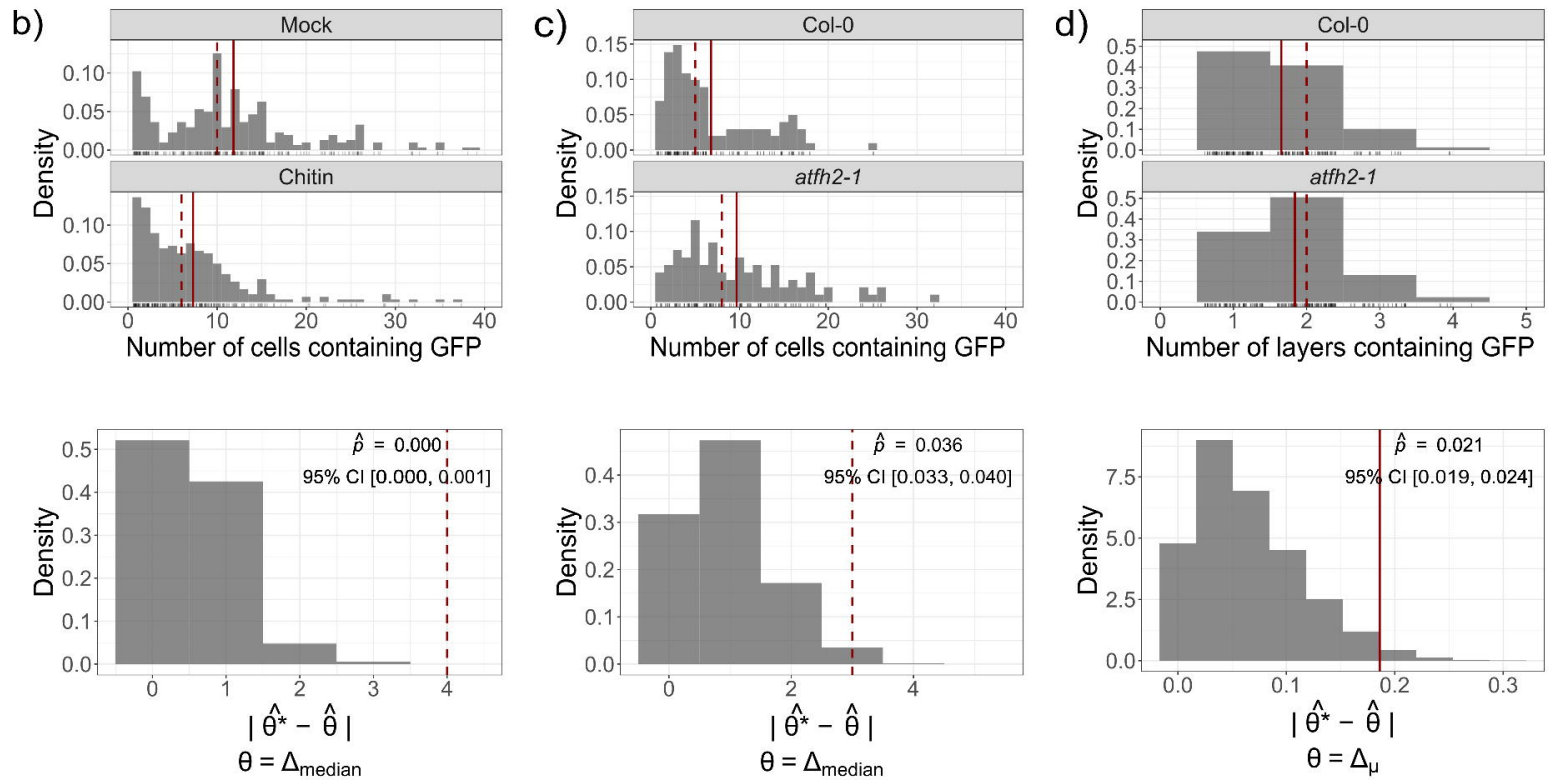